\title{
Caracterización Histológica y Morfológica del Tracto Digesti- vo de la Merluza Austral (Merluccius australis)
}

\author{
Histological and Morphological Characterization of the \\ Digestive Tract of Southern Hake (Merluccius australis)
}

\author{
Edison Serrano*; Camila Leiva**; Carlos Lonza*; Juan Carlos Sánchez*; \\ Jurij Wacyk $^{* * * *}$; Tatiana Zegers ${ }^{* * *} \&$ Marcos Godoy ${ }^{* * *}$
}

\begin{abstract}
SERRANO, E.; LEIVA, C.; LONZA, C.; SÁNCHEZ, J. C; WACYK, J.; ZEGERS, T. \& GODOY, M. Caracterización histológica y morfológica del tracto digestivo de la merluza austral (Merluccius australis). Int. J. Morphol., 32(3):1079-1084, 2014.

RESUMEN: Las características anatómicas e histológicas del tracto alimentario de la merluza austral (Merluccius australis) se han estudiado con el fin de comprender la fisiología digestiva y formular alimentos balanceados adecuados. M. australis posee un esófago corto, un estómago en forma de y grande y un intestino corto. Histológicamente, el tracto digestivo está formado por cuatro capas: la mucosa, submucosa, muscularis y la serosa. El esófago es un órgano tubular corto de pared gruesa con una gran capacidad de distensión y abundantes células caliciformes secretoras de mucus que permite el paso de grandes presas. El estómago es grande y musculoso, con alta capacidad de distensión. No se observo la presencia de apéndices pilóricos. El intestino es corto y dividido en regiones: proximal, medio y distal. En la región anterior del intestino se observaron abundantes células caliciformes, que disminuyeron en la región media para finalmente dispersarse en la región posterior. Además, los enterocitos mostraron un aumento en la presencia de vacuolas supranuclear en la región posterior del intestino. Los resultados de este estudio anatómico e histológico indican que M. australis tiene un sistema digestivo similar a otros peces gadiformes, el cual está adaptado para digerir y absorber presas ricas en proteínas, principalmente pescados y crustáceos.
\end{abstract}

PALABRAS CLAVE: Merluccius australis; Merluza austral; Tracto digestivo; Histología; Morfología intestinal.

\section{INTRODUCCIÓN}

En Chile, la salmonicultura constituye el rubro productivo de mayor volumen dentro de las exportaciones acuícolas (Wurmann, 2007). No obstante, en los últimos años se ha generando una gran incertidumbre y, en algunos casos, cambios en la estructura de la industria productora de salmones en Chile debido a los comportamientos variables de la demanda, los bajos precios de los productos tradicionales derivados de esta industria y como resultado de las crisis sanitarias producidas por el virus ISA y la caligidosis (Asche et al., 2009; Bravo et al., 2013; Godoy et al., 2013; Soluri, 2011).

Como consecuencia de lo anterior, en la última década, se ha desarrollado un creciente interés público y privado por invertir en investigación y desarrollo con el fin de diversificar la producción acuícola nacional, utilizando como plataforma la infraestructura de la industria del salmón (Silva, 2011). Dentro de las especies candidatas para diversifi- car la acuicultura nacional se encuentra la merluza austral (Merluccius australis) (Rosenlund \& Skretting, 2006). Esta especie demersal bentopelágica de hábitos carnívoros alcanza una longitud promedio de $65 \mathrm{~cm}$ y se encuentra desde la latitud $35^{\circ}$ L.S al extremo sur del país entre los 60 y 500 metros de profundidad desarrollándose a temperaturas entre los $3,8^{\circ} \mathrm{C}$ y $12^{\circ} \mathrm{C}$ (Aguayo-Hernandez, 1995; Gorini et al., 2010). Actualmente, la captura de M. australis ha demostrado tener una gran importancia comercial siendo sus principales mercados de destino España, Portugal, República Popular de China, Japón, Estados Unidos de América, Italia y Alemania (Sylvia, 1995), en donde alcanza precios promedios de 7,5 dólares el kilo (Mercabarna, 2012).

El desarrollo del cultivo de M. australis se ha visto enfrentada a diversas brechas científico-tecnológicas, debido a que aún se desconocen muchos aspectos biológicos básicos de la especie (Rosenlund \& Skretting). Uno de los

* Estación Experimental Quillaipe, Unidad de gestión tecnológica, Área de Alimentos y Biotecnología, Fundación Chile, Puerto Montt, Chile.

** Centro de Investigaciones Biológicas Aplicadas (CIBA), Puerto Montt, Chile.

**** Departamento de producción Animal, Facultad de Ciencias Agronómicas, Universidad de Chile, Santiago, Chile. 
aspectos determinantes en el éxito de cualquier cultivo de peces es el manejo y conocimiento de la alimentación y nutrición de la especie a cultivar (Cahu, 2004; De Silva et al., 2012).

Tradicionalmente, una de las metodologías básicas para comenzar a estudiar los requerimientos nutricionales de los peces silvestres es a través de la determinación de las preferencias alimenticias de la especie de interés mediante la caracterización de los componentes de su dieta natural y el valor nutricional de estos (Amezaga, 1988). No obstante, aun cuando estas metodologías entregan información relevante al momento de establecer las bases nutricionales de una especie, es necesario complementarla con otros estudios más específicos.

Diversos autores han demostrado que el estudio de las variaciones en las estructuras anato-morfológicas del sistema digestivo de las especies de peces a cultivar son necesarias para orientar el desarrollo de investigaciones en el área de la nutrición, tales como la preparación de dietas y el manejo de la alimentación (Al-Hussaini, 1949; Buddington et al., 1997; Wilson \& Castro, 2010).

Teniendo esto en mente, el presente estudio tiene como finalidad el caracterizar histológica y morfológicamente el sistema digestivo de la merluzaaustral (M. australis) permitiendo aportar información básica para el desarrollo y evaluación de alimentos formulados industrialmente.

\section{MATERIAL Y MÉTODO}

Colección de peces. Doce ejemplares de M. australis fueron colectados en la bahía de Quillaipe (latitud longitud) a una profundidad de entre 250 y $300 \mathrm{~m}$. La captura de los ejemplares fue realizada mediante el uso de espineles, los cuales fueron ascendidos en un tiempo aproximado de 30 minutos. Cada ejemplar de merluza austral al ser extraída se sacrificó mediante un golpe en la cabeza y posteriormente se etiqueto un microchip para su identificación al momento de colectar las muestras en el laboratorio de Necropsias de Fundación Chile Quillaipe.

Muestreo de órganos. De cada una de los individuos capturados se obtuvo muestras de esófago, estómago, intestino anterior, medio y posterior. Las muestras de tejidos se limpiaron cuidadosamente con una solución salina fisiológica para eliminar todo el contenido de alimento de los peces y posteriormente se cortaron en secciones longitudinales de $0,5 \mathrm{~cm}$ usando un bisturí estéril.
Análisis histológicos. Las muestras fueron fijadas en una solución de formalina al $4 \%$ buffer fosfato durante 24 horas para posteriormente ser sumergidas en una solución de alcohol al 70\%. Luego que las muestras histológicas fueron fijadas, estas fueron deshidratadas, incluidas enparafina y cortadas en secciones de aproximadamente 5 micras. Las secciones fueron teñidas con $\mathrm{H} \& \mathrm{E}$, para luego ser examinadas por microscopía de luz y fotografiadas digitalmente.

\section{RESULTADOS}

Descripción macroscópica. De las muestras analizadas se puede decir que la merluza Austral (M. australis) presenta un canal alimentario dividido en boca, esófago, estomago, intestino y ano, además de las órganos digestivos (hígado y páncreas).

La boca se encuentra ubicada de manera terminal y carece de barbas maxilares. Su tamaño es relativamente grande en proporción con la cabeza, presentando dientes mandibulares largos y afilados en los bordes mandíbulares y maxilares y dientes bucales en el techo, paredes laterales y suelo de lacavidad bucofaríngea además de presentar esta estructuras en la lengua.

M. australis presenta un esófago tubular, corto y musculoso, el cual que se extiende desde el extremo posterior de la faringe a la región cardíaca anterior del estómago. El estómago se observa como un saco muscular que presenta forma de $\mathrm{Y}$, el cual se divide en tres regiones: cárdica, fúndica y pilórica. No se observaron ciegos pilóricos definidos. El intestino de merluza austral es moderadamente largo y dividido en tres regiones: anterior, media y posterior. En relación a la disposición del intestino en la cavidad abdominal, se observo que este órgano se extiende hacia delante por una corta distancia donde se curva fuertemente hacia atrás hacia la porción cardiaca de estómago. Posteriormente al alcanzar la región media de la cavidad abdominal, el intestino se curva hacia adelante en dirección de la región fúndica del estomago, donde nuevamente se curva en la dirección contraria hacia el recto. Ventralmente, el recto termina en el ano, situado frente a la aleta anal.

Descripción microscópica. En general, los diferentes órganos que componen el tracto digestivo de merluza austral presentaron cuatro capas constitutivas: mucosa, submucosa, muscular y serosa.

Esófago. Esta estructura presentó una capa mucosa que además de presentar numerosos pliegues, posee un epitelio estratificado con abundante cantidad de células mucosas de 
diferente tamaño. Bajo este tejido se encuentra la submucosa que en este caso está formada por tejido conectivo denso con una gran cantidad de fibroblastos. De forma más externa se observa la capa muscular de gran desarrollo en un corte transversal se distinguen dos capas de musculo estriado una capa externa de fibras musculares circulares y una capa interna de musculo con fibras longitudinales, que se extienden en la submucosa. La distribución de las distintas estructuras histologicas se observa en la Figura 1.

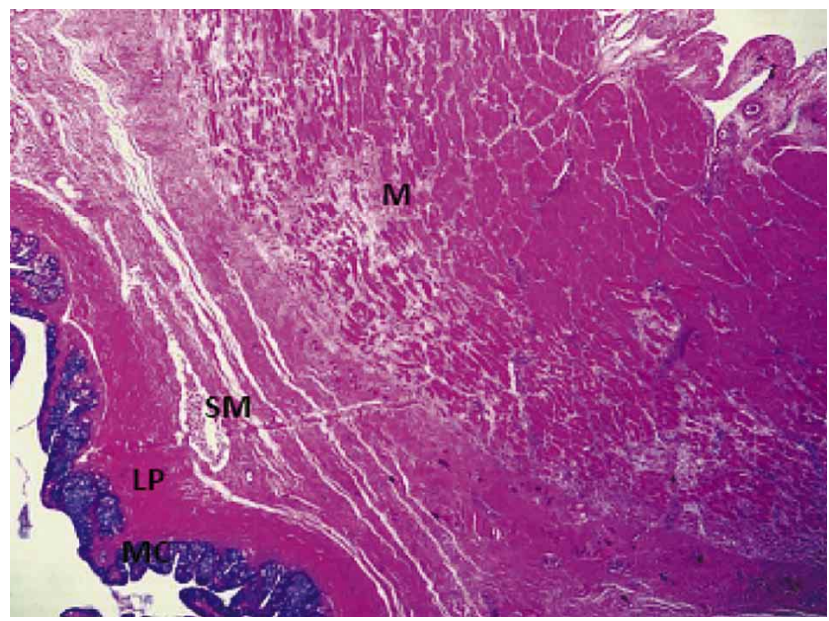

Fig. 1. Esófago de M. australis (H\&E 40X). Se observa la mucosa (MC) con epitelio estratificado, se observan numerosos pliegues y abundante cantidad de células mucosas. La lamina propia (LP) formada por epitelio estratificado. Submucosa (SM) formada por tejido conectivo laxo). Gruesa capa muscular (M) formada por fibras musculares circulares externa y fibras musculares longitudinales interna

Estómago. Esta estructura presentó un lumen de gran tamaño en relación al diámetro de los otros órganos evaluados. La mucosa presenta epitelio columnar plegado con células mucosas cuyo núcleo se ubica en la región basal de la célula y el citoplasma con contenido mucoso en la región apical. En la porción baja de los pliegues gástricos se encuentran las glándulas gástricas. La capa muscular de la mucosa es delgada. La submucosa presentó numerosos vasos sanguíneos. La capa muscular externa a la sub-mucosa es gruesa formada por dos capas: una interna más gruesa con fibras longitudinales y una capa externa más delgada con fibras circulares. La distribución de las distintas estructuras histológicas del estomago se observa en la Figura 2.

Intestino. La capa mucosa mas interna en el intestino presento una organización en forma de pliegues o vellosidades ramificadas formados por el epitelio columnar y una delgada lámina propia. El estrato compacto y el estrato granuloso se localizan debajo de estos pliegues.

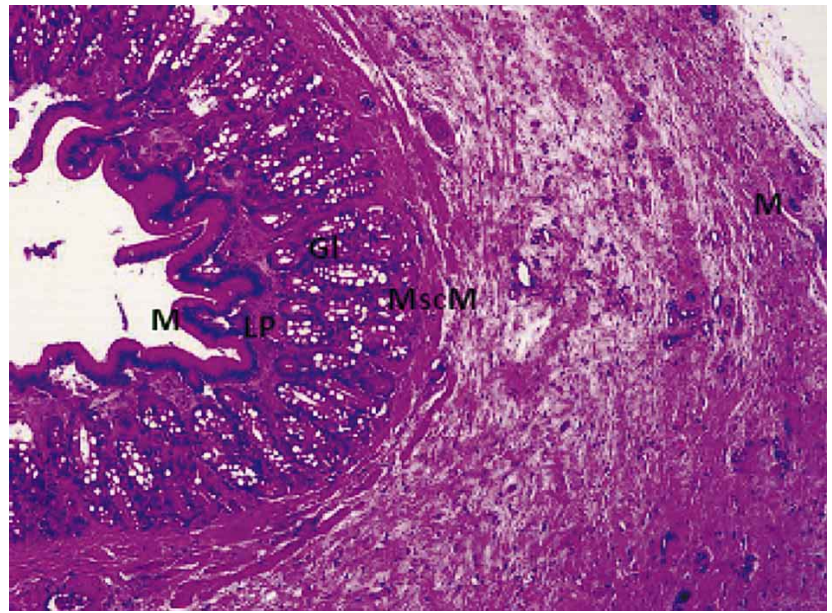

Fig. 2. Estomago de M. australis (H\&E 100X). Se observa la mucosa (M) con epitelio simple plegado con células mucosas, lámina propia (LP) con numerosas glándulas gástricas $(\mathrm{Gl})$, capa muscular de la mucosa (MscM). Capa muscular (M) con una capa interna formada por fibras longitudinales y una interna formada por fibras circulares.

En el segmento anterior del intestino se observó un mayor número y tamaño de los pliegues, los cuales disminuyen progresivamente hacia la región posterior.

Del mismo modo, la cantidad de células mucosas en el epitelio columnar varía a lo largo del intestino. En la porción anterior se observa una abundante cantidad de células mucosas de gran tamaño, las cuales decrecen en la porción media para finalmente dispersarse en la región posterior (Figs. 3, 4 y 5). Los enterocitos, por su parte, presentan un

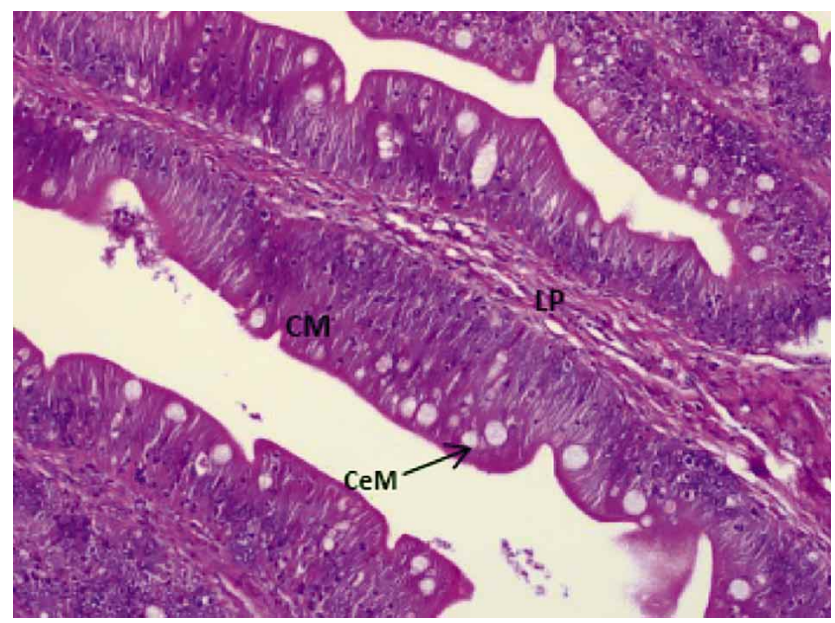

Fig. 3. Intestino anterior de M. australis (H\&E, 200X). Se observa en las vellosidades intestinales la capa mucosa (CM) formada por epitelio columnar, con presencia de escasa cantidad de células mucosas (CeM) de gran tamaño. Se observa una delgada lámina propia (LP). 
aumento en la presencia de vacuolas supranucleares desde la región anterior hacia la región posterior del intestino.

Finalmente, la capa submucosa está constituida en esta sección del tracto digestivo de tejido conectivo laxo con numerosos vasos sanguíneos, mientras que la capa muscular es delgada y está formada por músculo liso en dos orientaciones: una interna más gruesa con fibras circulares y una externa más delgada con fibras longitudinales.

La distribución de las distintas estructuras histológicas de las regiones anterior, media y posterior se observan en las Figuras 4, 5 y 6, respectivamente.

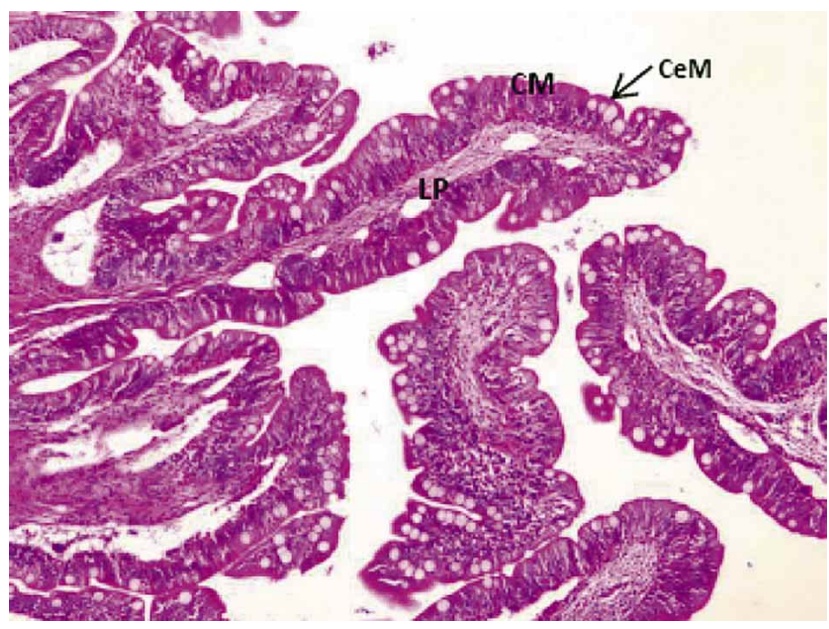

Fig. 4. Intestino medio de M. australis (H\&E, 200X).Se observa en vellosidades intestinales la capa mucosa $(\mathrm{CM})$ formada por epitelio columnar, con presencia de moderada cantidad de células mucosas $(\mathrm{CeM})$ de mediano tamaño. Se observa una delgada lámina propia (LP) en las vellosidades.

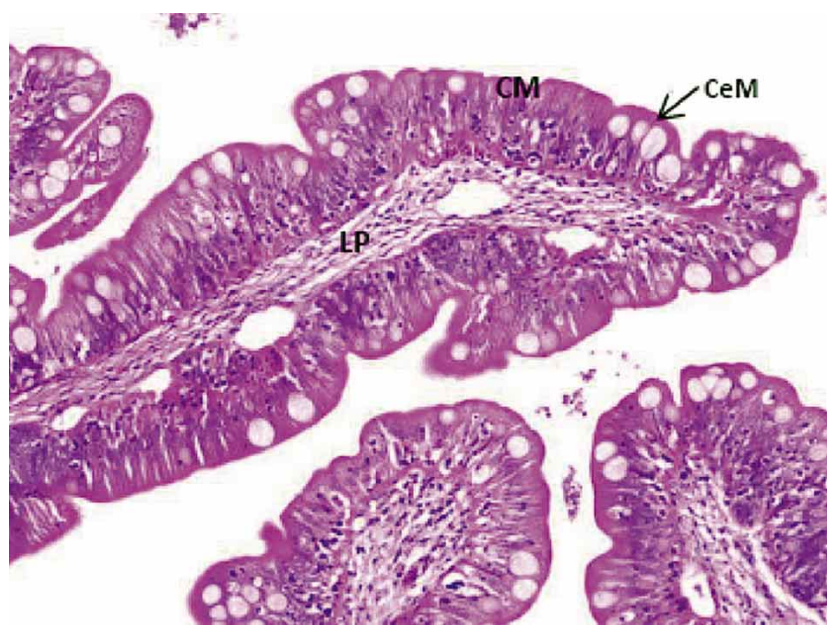

Fig. 5. Intestino posterior de M. australis (H\&E, 200 X).Se observa en vellosidades intestinales la capa mucosa $(\mathrm{CM})$ formada por epitelio columnar, con presencia de abundante cantidad de células mucosas $(\mathrm{CeM})$ de mediano tamaño. Se observa una delgada lámina propia (LP) en las vellosidades.

\section{DISCUSIÓN}

La descripción del tracto digestivo de la merluza austral proporciona información valiosa en relación a la anatomía, digestiva y hábitos alimenticios de esta especie. M. australis presenta un sistema digestivo típico de un pez pelágico carnívoro ictiófago. Su aparato digestivo presenta las adaptaciones típicas a un régimen alimentario carnívoro y predador caracterizado por un gran estomago y un intestino relativamente corto (Buddington et al.; Forde-Skjaervik et al., 2006; Hellberg \& Bjerkas, 2000; Kuzir et al., 2012; Lokka et al., 2013). Histológicamente, el tracto digestivo de merluza austral muestra una conformación tisular similar a la reportada para otros teleósteos, con cuatro capas o túnicas constitutivas; mucosa, submucosa, muscular y serosa (Wilson \& Castro).

El esófago de esta especie es similar a la de otros teleósteos carnívoros, musculoso y con abundante células caliciformes secretoras de mucus (Buddington et al.; Hellberg \& Bjerkas; Lokka et al.), las cuales cumplen la función de lubricación y protección de la mucosa contra la abrasión física al momento de ingerir las presas. La abundancia de este tipo de células es característico de peces que se alimentan de cangrejos y otras presas con partes duras (Buddington et al.). Esto concuerda con estudios sobre los hábitos alimenticios de $\mathrm{M}$.australis en donde se observo que al menos un $10 \%$ de la dieta de esta especie se basaba en diversos crustáceos además de otras especies de peces (Aguayo-Hernandez).

M. australis presenta un estómago de gran tamaño y musculoso con gran capacidad de distención, característico de especies de peces que tragan presas enteras de gran tamaño. La disposición de la capa muscular en dos orientaciones permite una adecuada mezcla con los jugos gástricos así como la generación de partículas de menor tamaño mejorando la digestión y absorción de nutrientes por parte del intestino al facilitar la disrupción física del alimento y la mezcla con las secreciones gástricas (Buddington et al.). Esta clase de distribución de las fibras musculares en la capa muscular del estómago también hasido observada en otras especies de peces carnívoros tales como Salmo salar (Lokka et al.), Esoxlucius (Bucke, 1971), Anguilla anguilla (Clarke \& Witcomb), Anarhichas lupus (Hellberg \& Bjerkas) y Hippoglossus hippoglossus.

El intestino de $M$. australis difiere morfológicamente de otras especies de peces carnívoros debido a quecarecede ciegos pilóricos. La ausencia de ciegos pilóricos ha sido descrita como una característica en 
todos los miembros de la familia merlucidae (Cohen et al., 1990). Estas estructuras anatómicas ayudan a los peces carnívoros a aumentar la superficie de absorción y asimilación de nutrientes, compensando de esta forma la corta longitud del intestino (Bakke et al., 2010; Buddington et al.; Wilson \& Castro). Ante la carencia de los ciegos pilóricos, M. australis compensaría la función de esta estructura mediante una mayor actividad de micropinocitosis proteica en la región posterior del intestino, en donde se observo una mayor cantidad de vacuolas supranucleares en los enterocitos. Similares resultados fueron descritos en M. merluccius por Kozaric et al. (2004), quienes reportaron la presencia de vacuolas densas con proteínas en los enterocitos del intestino posterior, lo cual estaría correlacionado con una mayor actividad de las enzimas fosfatasas acida y alcalina, demostrando que esta región del tracto digestivo es donde ocurrenlos principales procesos de absorción de nutrientes. No obstante, esta estrategia fisiológica difiere lo reportado generalmente enteleósteos, en donde la absorción de nutrientes ocurre principalmente en el intestino anterior y ciegos pilóricos (Bakke et al.; Buddington et al.).

En conclusión, M. australis es un pez carnívoro con tracto digestivo adaptado para digerir y absorber presas ricas en proteínas, principalmente peces y crustáceos.Por lo cual,el tipo de alimento que tendría que entregarse a esta especie bajo condiciones de cultivo debe considerar niveles altos de proteína, probablemente de origen marino y bajos niveles de lípidos en su formulación y elaboración.

\section{AGRADECIMIENTOS}

Los autores agradecen el financiamiento otorgado por CONICYT a través del proyecto FONDEF DA09I 1001 "Optimización e innovación tecnológica para el cultivo de la Merluza Austral (Merluccius australis): Captura, aclimatación y acondicionamiento de reproductores".

SERRANO, E.; LEIVA, C.; LONZA, C.; SÁNCHEZ, J. C; WACYK, J.; ZEGERS, T. \& GODOY, M. Histological and morphological characterization of the digestive tract of southern hake (Merluccius australis). Int. J. Morphol., 32(3):1079-1084, 2014.

SUMMARY: The anatomy and histology features of alimentary tract of southern hake (Merluccius australis) have been studied in order to understand the digestive physiology and formulate suitable feeds. M. australis has a short oesophagus, large yshaped stomach and short intestine, and histologically, the digestive tract is formed by four layers: mucosa, submucosa, muscularis and serosa. The oesophagus is a short tubular thick-walled organ with a great distension capacity and abundant mucus secreting goblet cells allowing the passage of large preys. The stomach is large and muscular with high capability for distension. Pyloric caeca were not present. The intestine is short and divided into a proximal middle and distal segment. In the anterior region of the intestine was observed abundant goblet cells, which decreased in the middle region and finally dispersed in the posterior region. Additionally, enterocytes showed an increase in the presence of supranuclear vacuoles at the posterior region of the intestine. The results of this anatomical and histological study indicate that $M$. australis has a digestive tract similar to other gadiform fishes, which is adapted to digest and absorb protein-rich preys, mainly fish and crustacean.

KEY WORDS: Merluccius australis; Southern hake; digestive tract; histology; intestinal morphology.

\section{REFERENCIAS BIBLIOGRÁFICAS}

Aguayo-Hernández, M. Biology and fisheries of Chilean hakes (M. gayi and M. australis). En: Alheit, J. \& Pitcher, T. (Eds.). Hake: Biology, Fisheries and Markets. London, Chapman \& Hall, 1995. pp.305-37.

Al-Hussaini, A. H. On the functional morphology of the alimentary tract of some fish in relation to differences in their feeding habits; anatomy and histology. Q. J. Microsc. Sci., 90 Pt. 2(2):109-39, 1949.

Amezaga, R. Análisis de contenidos estomacales en peces. Revisión bibliográfica de los objetivos y la metodología. Inf. Téc. Inst. Esp. Oceanogr., 63:1-74, 1988.
Asche, F.; Hansen, H. V.; Tveteras, R. \& Tveteras, S. R. The salmon disease crisis in Chile. Mar. Resour. Econ., 24:405-11, 2009.

Bakke, A. M.; Glover, C. \& Krogdahl, A. Feeding, digestion and absorption of nutrients. En: Grosell, M.; Farrell, A. P. \& Brauner, C. J. (Eds.). Fish Physiology Volume 30. The multifunctional gut of fish. San Diego, Academic Press, 2010. pp.57-110.

Bravo, S.; Nuñez, M. \& Silva, M. T. Efficacy of the treatments used for the control of Caligus rogercresseyi infecting Atlantic salmon, Salmo salar L., in a new fish-farming location in Region XI, Chile. J. Fish Dis., 36(3):221-8, 2013. 
Bucke, D. The anatomy and histology of the alimentary tract of the carnivorous fish the pike Esox lucius L. J. Fish Biol., 3(4):421-31, 1971.

Buddington, R. K.; Krogdahl, A. \& Bakke-McKellep, A. M. The intestines of carnivorous fish: structure and functions and the relations with diet. Acta Physiol. Scand. Suppl., 638:67-80, 1997.

Cahu, C. Domestication and nutrition in fish. Prod. Anim., 17(3):205-10, 2004.

Clarke, A. J. \& Witcomb, D. M. A study of the histology and morphology of the digestive tract of the common eel (Anguilla anguilla). J. Fish Biol., 16:159-70, 1980.

Cohen, D. M.; Inada, T.; Iwamoto, T. \& Scialabba, N. FAO Species Catalogue. Vol.10. Gadiform fishes of the world (Order Gadiformes). An annotated and illustrated catalogue of cods, hakes, grenadiers and other gadiform fishes known to date. FAO Fisheries Synopsis. No. 125, Vol. 10. Rome, FAO, 1990.

De Silva, S.; Turchini, G. \& Francis, D. Nutrition. En: Lucas, J. S. \& Southgate, P. C. (Eds.). Aquaculture-Farming Aquatic Animals and Plants, 2nd ed. Oxford, Wiley-Blackwell, 2012. pp.164-87.

Forde-Skjaervik, O.; Refstiea, S.; Aslaksena, M. A. \& Skrede, A. Digestibility of diets containing different soybean meals in Atlantic cod (Gadus morhua); comparison of collection methods and mapping of digestibility in different sections of the gastrointestinal tract. Aquac., 261(1):241-58, 2006.

Godoy, M. G.; Kibenge, M. J.; Suarez, R.; Lazo, E.; Heisinger, A.; Aguinaga, J.; Bravo, D.; Mendoza, J.; Llegues, K. O.; Avendaño-Herrera, R.; Vera, C.; Mardones, F. \& Kibenge, F. $\mathrm{S}$. Infectious salmon anaemia virus (ISAV) in Chilean Atlantic salmon (Salmo salar) aquaculture: emergence of low pathogenic ISAV-HPR0 and re-emergence of virulent ISAVHPRA: HPR3 and HPR14. Virol. J., 10:344, 2013.

Gorini, F. L.; Abachian, V. E. \& Giussi, A. R. Age and growth of southern hake Merluccius australis (Hutton, 1872) of the Argentine Sea. Rev. Invest. Desarr. Pesq., (20):65-73, 2010.

Hellberg, H. \& Bjerkas, I. The anatomy of the oesophagus, stomach and intestine in common wolffish (Anarhichas lupus L.): a basis for diagnostic work and research. Acta Vet. Scand., 41(3):283-97, 2000

Kozaric, Z.; Kuzir, S.; Nejedli, S.; Petrinec, Z. \& Sreboèan, E. Histochemical distribution of digestive enzymes in hake, Merluccius merluccius L. 1758. Vet. Arhiv., 74(4):299-308, 2004.

Kuzir, S.; Gjurcevic, E.; Nejedli, S.; Bazdaric, B. \& Kozaric, Z. Morphological and histochemical study of intestine in wild and reared European eel (Anguilla anguilla L.). Fish Physiol. Biochem., 38(3):625-33, 2012.
Lokka, G.; Austbø, L.; Falk, K.; Bjerkss, I. \& Koppang, E. O. Intestinal morphology of the wild Atlantic salmon (Salmo salar). J. Morphol., 274(8):859-76, 2013.

Rosenlund, G. \& Skretting, M. Worldwide status and perspective on gadoid culture. ICES J. Mar. Sci., 63(2):194-7, 2006.

Silva, K. En Chile: Estado del potenciales especies para diversificar la acuicultura. Mundo acuícola, 82:6-8, 2011.

Soluri, J. Something fishy: Chile's blue revolution, commodity diseases, and the problem of sustainability. Lat. Am. Res. Rev., 46:55-81, 2011.

Sylvia, G. Global markets and products of hake. En: Alheit, J. \& Pitcher, T. (Eds.). Hake: Biology, Fisheries and Markets. London, Chapman \& Hall, 1995. pp.415-35.

Wilson, J. M. \& Castro, L. F. C. Morphological diversity of the gastrointestinal tract in fishes. En: Grosell, M.; Farrell, A. P. \& Brauner, C. J. (Eds.). Fish Physiology Volume 30. The multifunctional gut of fish. San Diego, Academic Press, 2010. pp.1-55.

Wurmann, C. F. Salmon Farming in Chile: History, Policies, and Development Strategies. En: Leung, P. S.; Lee, C. S. \& O'Bryen, P. J. (Eds.). Species and System Selection for Sustainable Aquaculture. Oxford, Wiley-Blackwell, 2007. pp.415-44.

\section{Dirección para correspondencia: \\ Dr. Edison Serrano \\ Estación Experimental Quillaipe \\ Unidad de gestión tecnológica \\ Área de Alimentos y Biotecnología \\ Fundación Chile \\ Puerto Montt \\ CHILE}

Email: erserrano@gmail.com

Recibido : 11-03-2014

Aceptado: 22-07-2014 